\title{
Laser damage of free-standing nanometer membranes
}

\author{
Yuya Morimoto, ${ }^{1,2}$ Iännis Roland, ${ }^{3}$ Stéphanie Rennesson, ${ }^{4}$ Fabrice Semond, ${ }^{4}$ \\ Philippe Boucaud, ${ }^{3}$ and Peter Baum ${ }^{1,2, a)}$ \\ ${ }^{1}$ Ludwig-Maximilians-Universität München, Am Coulombwall 1, 85748 Garching, Germany \\ ${ }^{2}$ Max-Planck-Institute of Quantum Optics, Hans-Kopfermann-Straße 1, 85748 Garching, Germany \\ ${ }^{3}$ Centre de Nanosciences et de Nanotechnologies, CNRS, Univ. Paris-Sud, Université Paris-Saclay, \\ Bâtiment 220, Rue André Ampère, F-91405 Orsay, France \\ ${ }^{4}$ Université Côte d' Azur, CRHEA-CNRS, Rue Bernard Grégory, F-06560 Valbonne, France
}

(Received 11 September 2017; accepted 7 November 2017; published online 4 December 2017)

\begin{abstract}
Many high-field/attosecond and ultrafast electron diffraction/microscopy experiments on condensed matter require samples in the form of free-standing membranes with nanometer thickness. Here, we report the measurement of the laser-induced damage threshold of 11 different free-standing nanometer-thin membranes of metallic, semiconducting, and insulating materials for 1-ps, 1030-nm laser pulses at $50 \mathrm{kHz}$ repetition rate. We find a laser damage threshold that is very similar to each corresponding bulk material. The measurements also reveal a band gap dependence of the damage threshold as a consequence of different ionization rates. These results establish the suitability of free-standing nanometer membranes for high-field pump-probe experiments. Published by AIP Publishing. https://doi.org/10.1063/1.5004081
\end{abstract}

\section{INTRODUCTION}

Free-standing ultrathin membranes are widely used in many types of ultrafast pump-probe investigations. For example, in attosecond electron diffraction, ${ }^{1}$ attosecond optical spectroscopy, ${ }^{2-4}$ and high-harmonic generation, ${ }^{5-8}$ sub$\mu$ m-membranes can avoid optical dispersion, linear/nonlinear absorption, and other pulse propagation effects, which are critical for single-cycle or few-cycle excitation, and therefore provide the clearest spectral results. The nanometer-thick membranes are also essential for studying free-electron quantum optics ${ }^{9}$ and for performing ultrafast transmission electron microscopy and diffraction, ${ }^{10-12}$ techniques that can record atomic and electronic motions in space and time. ${ }^{13-19}$ Here, the sample must be thin enough, typically less than $100 \mathrm{~nm}$, in order to let electrons pass without too much absorption losses or inelastic scattering. Freestanding membranes are also crucial for electron pulse compression and metrology with $\mathrm{THz}$ fields ${ }^{18,20}$ or attosecond electron microscopy and diffraction ${ }^{1}$ of light-driven electron dynamics. ${ }^{21}$ In all these solid-state experiments, the applicable laser field strength and therefore the most complex and efficient physics that can be initiated are usually limited by laser-induced damage and ultimately destruction of the membrane. Extensive knowledge about laser damage in ultrathin membranes is therefore a prerequisite for designing and advancing those kinds of experiments.

Most available laser damage reports focus on bulk materials or optical coatings on substrates, ${ }^{22-26}$ and comprehensive studies of how free-standing ultrathin membranes are damaged by ultrashort laser pulses are rather scarce. In this work, we report such data for 11 different membranes of various metallic, semiconducting and insulating materials. We find that the applicable field strength increases with the band gap

a)peter.baum@1mu.de and that the nanometer membranes have no largely different damage thresholds than the corresponding bulk materials. These results establish the high prospect of nanometer-thin membranes for attosecond/high-field spectroscopy, electron diffraction, and other types of investigations, and the reported relationships and damage values should be useful for designing future experiments.

\section{BACKGROUND}

Ultrathin membranes, in the context of this work, shall be membranes with less than $1 \mu \mathrm{m}$ thickness and of many $\mu \mathrm{m}$ in lateral size, i.e., big enough to let an excitation laser beam pass through without edge effects. Free-standing membranes are usually suspended over a metal mesh or over a hole in a substrate, and both surfaces are exposed to air or vacuum. With respect to laser damage, such ultrathin free-standing membranes typically have some detrimental features which are not present in bulk materials: (i) rather low heat removal, (ii) weakness to mechanical stress, (iii) some potentially strong optical interference, and (iv) relatively low material quality. Substantial heat transfer is only possible along the plane of the membranes, ${ }^{27}$ and therefore, the removal of heat deposited by repetitive laser pulses is inefficient, in particular, at higher laser repetition rates that are desired in ultrafast electron diffraction/microscopy in order to avoid space charge broadening of the electron pulses. Free-standing membranes are weak to mechanical stress, and a tiny crack or little melting/ablation can quickly lead to macroscopic destruction. Inside a dielectric or semiconducting free-standing membrane, the incident and reflected laser electric fields interfere with each other. The maximum electric field amplitude is often located at the rear surface, where the constructive interference occurs. The typical preparation processes are complex and can involve thin-film growth followed by etching/ transfer, thinning of a macroscopic material by ion beams or 
TABLE I. Free-standing membranes and their laser-induced damage thresholds for 1-ps pulses. The values of $F_{50 \mathrm{kHz}}$ and $F_{\text {burst }}$ represent the laser damage thresholds measured without and with the fast optical chopper (see Fig. 1), respectively.

\begin{tabular}{|c|c|c|c|c|c|}
\hline Material & Thickness (nm) & Band gap (eV) & $F_{50 \mathrm{kHz}}\left(\mathrm{J} / \mathrm{cm}^{2}\right)$ & $F_{\text {burst }}\left(\mathrm{J} / \mathrm{cm}^{2}\right)$ & $F_{\text {burst }} / F_{50 \mathrm{kHz}}$ \\
\hline $\mathrm{Cu}$ & 35 & 0 & $(9.8 \pm 1.2) \times 10^{-3}$ & $(1.5 \pm 0.2) \times 10^{-2}$ & $1.5 \pm 0.3$ \\
\hline $\mathrm{Au}$ & 30 & 0 & $(6.3 \pm 1.2) \times 10^{-2}$ & $(9.8 \pm 1.1) \times 10^{-2}$ & $1.5 \pm 0.4$ \\
\hline Graphene & Monolayer & 0 & $(1.2 \pm 0.4) \times 10^{-2}$ & $(2.0 \pm 1.1) \times 10^{-2}$ & $1.7 \pm 1.1$ \\
\hline $\mathrm{Ge}$ & 100 & 0.6 (Ref. 43) & $(7.7 \pm 1.1) \times 10^{-3}$ & $(9.0 \pm 1.5) \times 10^{-3}$ & $1.2 \pm 0.3$ \\
\hline $\mathrm{Si}$ & 35 & 1.2 (Ref. 44) & $(5.3 \pm 0.6) \times 10^{-2}$ & $(5.9 \pm 0.7) \times 10^{-2}$ & $1.1 \pm 0.2$ \\
\hline $\mathrm{TiO}_{2}$ & 150 & 3.6 (Ref. 45) & $(2.3 \pm 0.4) \times 10^{-1}$ & $(2.5 \pm 0.4) \times 10^{-1}$ & $1.1 \pm 0.3$ \\
\hline $\mathrm{Si}_{3} \mathrm{~N}_{4}$ & 50 & 4.8 (Ref. 46) & $(3.7 \pm 0.5) \times 10^{-1}$ & $(4.1 \pm 0.5) \times 10^{-1}$ & $1.1 \pm 0.2$ \\
\hline AlN & 80 & 6.2 (Ref. 47) & $(2.8 \pm 0.4) \times 10^{-1}$ & $(3.4 \pm 0.5) \times 10^{-1}$ & $1.2 \pm 0.3$ \\
\hline Formvar & $12-22$ & 6.3 (Ref. 48) & $1.9 \pm 0.3$ & $2.8 \pm 0.4$ & $1.4 \pm 0.3$ \\
\hline $\mathrm{Al}_{2} \mathrm{O}_{3}$ & 140 & 6.5 (Ref. 45) & $2.0 \pm 0.4$ & $1.6 \pm 0.3$ & $0.8 \pm 0.2$ \\
\hline $\mathrm{SiO}_{2}$ & 40 & 7.5 (Ref. 45) & $4.0 \pm 0.5$ & $4.3 \pm 0.8$ & $1.1 \pm 0.3$ \\
\hline
\end{tabular}

plasma etching, or single-layer cleaving techniques (Scotchtape). Therefore, free-standing membranes usually have a rather low flatness, a rough surface, and may contain substantial amount of impurities and crystallographic defects. To what extent these and other deficiencies are changing the laser damage threshold is the subject of this experimental report.

\section{EXPERIMENTAL SETUP AND MATERIALS}

We employed a Yb:YAG thin-disk regenerative amplifier, which delivered linearly polarized 1-ps pulses centered at $1030 \mathrm{~nm}$ at a repetition rate of $50 \mathrm{kHz} .{ }^{28}$ Laser damage thresholds were measured by continuously increasing the laser power on a $22 \times 32-\mu \mathrm{m}^{2}$ focus spot until each membrane was damaged, i.e., a "R-on-1 test." ${ }^{24}$ In Table I, we summarize the free-standing membranes that were investigated. Membranes of $\mathrm{Al}_{2} \mathrm{O}_{3}, \mathrm{Au}, \mathrm{Cu}, \mathrm{Ge}$, and $\mathrm{TiO}_{2}$ were formed on glass plates covered with betaine (1-carboxy- $N, N, N$-trimethylmethanammonium inner salt) by thermal $(\mathrm{Au}, \mathrm{Cu}, \mathrm{Ge})$ and electronbeam $\left(\mathrm{Al}_{2} \mathrm{O}_{3}, \mathrm{TiO}_{2}\right)$ evaporation. Afterwards, the betaine layer was dissolved in water, and the membranes were picked up by stainless-steel support plates which have 2-mm holes, thus creating free-standing membranes. The AlN membranes were produced by etching $40-\mu \mathrm{m}$ big holes into a $\mathrm{Si}$ (111) substrate on which AlN was epitaxially grown. ${ }^{29}$ There is a dislocation density of about $10^{10}$ per $\mathrm{cm}^{2}$, but the material is macroscopically single-crystalline, as confirmed by electron diffraction with a $100-\mu \mathrm{m}$ wide ultrafast beam. ${ }^{30}$ The membranes of formvar, graphene and $\mathrm{Si} / \mathrm{Si}_{3} \mathrm{~N}_{4} / \mathrm{SiO}_{2}$ were acquired from Plano $\mathrm{GmbH}$, TedPella, Inc., and SiMPore, Inc., respectively. The $\mathrm{Si}, \mathrm{Si}_{3} \mathrm{~N}_{4}$, and $\mathrm{SiO}_{2}$ membranes had a size of $100 \times 100 \mu \mathrm{m}^{2}$. The formvar membrane and graphene were suspended on gold grids with 300 and 2000 mesh/in., respectively. The Si and AlN membranes were single-crystalline, while all other membranes were probably more or less amorphous.

Figure 1(a) shows the experimental setup. Measurements were conducted in air. A linearly polarized 1-ps, 1030-nm laser beam at $50 \mathrm{kHz}$ repetition rate ${ }^{28}$ was focused onto each membrane by a $f=80 \mathrm{~mm}$ lens. The beam that impinged on the samples at normal incidence had a beam diameter at the focus of $22 \times 32 \mu \mathrm{m}^{2}$ (1/ $\mathrm{e}^{2}$ full width). A weak (less than 1 $\mathrm{mW}$ on sample) continuous-wave laser of wavelength $808 \mathrm{~nm}$ was used to probe the presence of damage in each membrane. The probe beam was focused on an area of $25 \times 13 \mu \mathrm{m}^{2}$ diameter on each sample from the opposite side. Transmittance of the probe beam with respect to a reference arm was measured with a balanced photodiode. By this method, we became sensitive to changes in transmittance by less than $0.1 \%$. The intensity of the 1-ps, 1030-nm excitation beam on the samples was controlled by a combination of a half-wave plate and a polarizing beam splitter. We scanned the power continuously
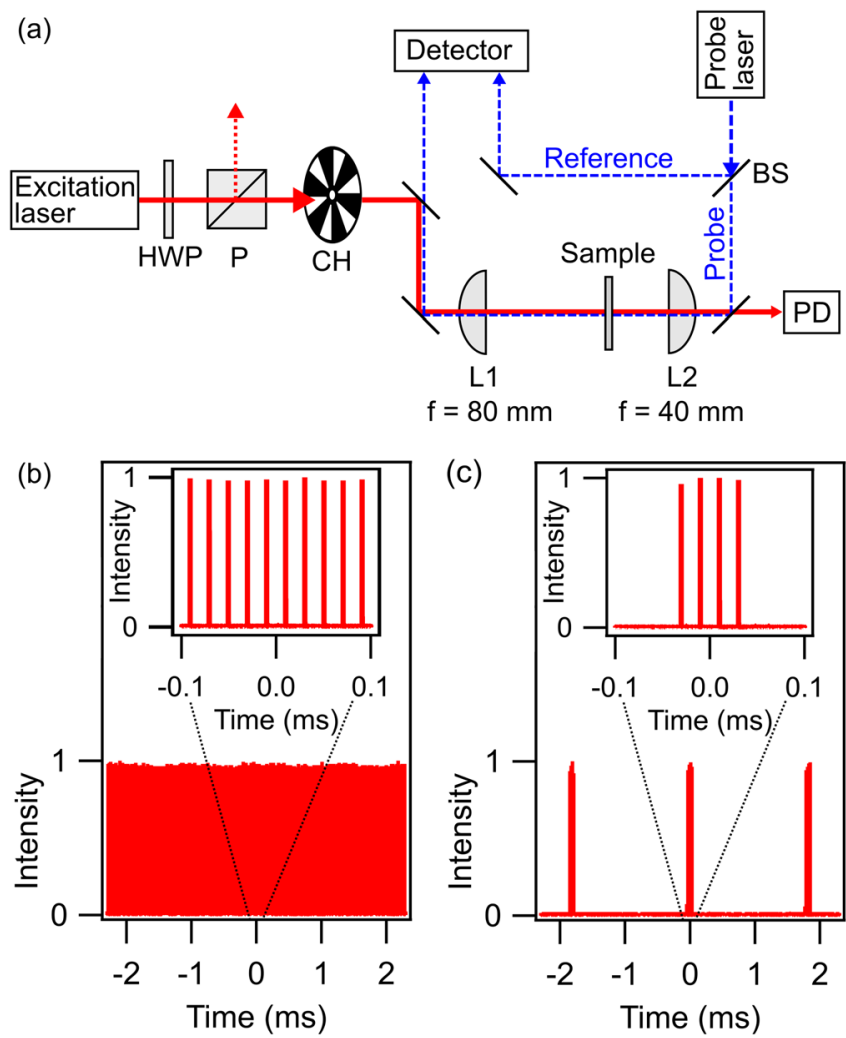

FIG. 1. Experimental methodology. (a) Setup for laser damage measurements of free-standing nanometer membranes. HWP, half-waveplate; P, polarizing beam splitter; $\mathrm{CH}$, optical chopper; L, lens; PD, photodiode; BS, beam splitter. (b) Measured photodiode output without the optical chopper. The excitation laser hits the sample at the $50 \mathrm{kHz}$ rate. (c) Photodiode signal with the fast optical chopper. The chopper transmits the excitation pulses at $1.8 \mathrm{~ms}$ intervals and reduces the average power to $5 \%$. Insets in (b) and (c) are the expanded graphs around a single pulse and show the short-time details. 
from zero to a power level well above the damage threshold, while continuously recording the transmittance of the probe beam. At each power level, the sample was exposed to more than $10^{6}$ laser shots, in order to mimic a continuous pumpprobe experiment under constant excitation conditions. We note that our laser damage methodology is also applicable to optical coatings and bulk materials, if there is enough transmission of the probe beam.

\section{RESULTS}

Figure 2 shows examples of these scans for $30 \mathrm{~nm}$ of $\mathrm{Au}$, $35 \mathrm{~nm}$ of $\mathrm{Si}$, and $150 \mathrm{~nm}$ of $\mathrm{TiO}_{2}$. In the cases of $\mathrm{Au}$ and $\mathrm{Si}$, the transmittance of the probe beam increases with a jump at well-defined thresholds (shown by arrows). Such an abrupt increase in transmittance is attributed to the formation of a macroscopic hole in the membrane. Indeed, after the experiment, holes were clearly seen by optical microscopy (see the insets). Above the threshold, transmittance increased further, indicating a growth of the hole size. The extremely sharp
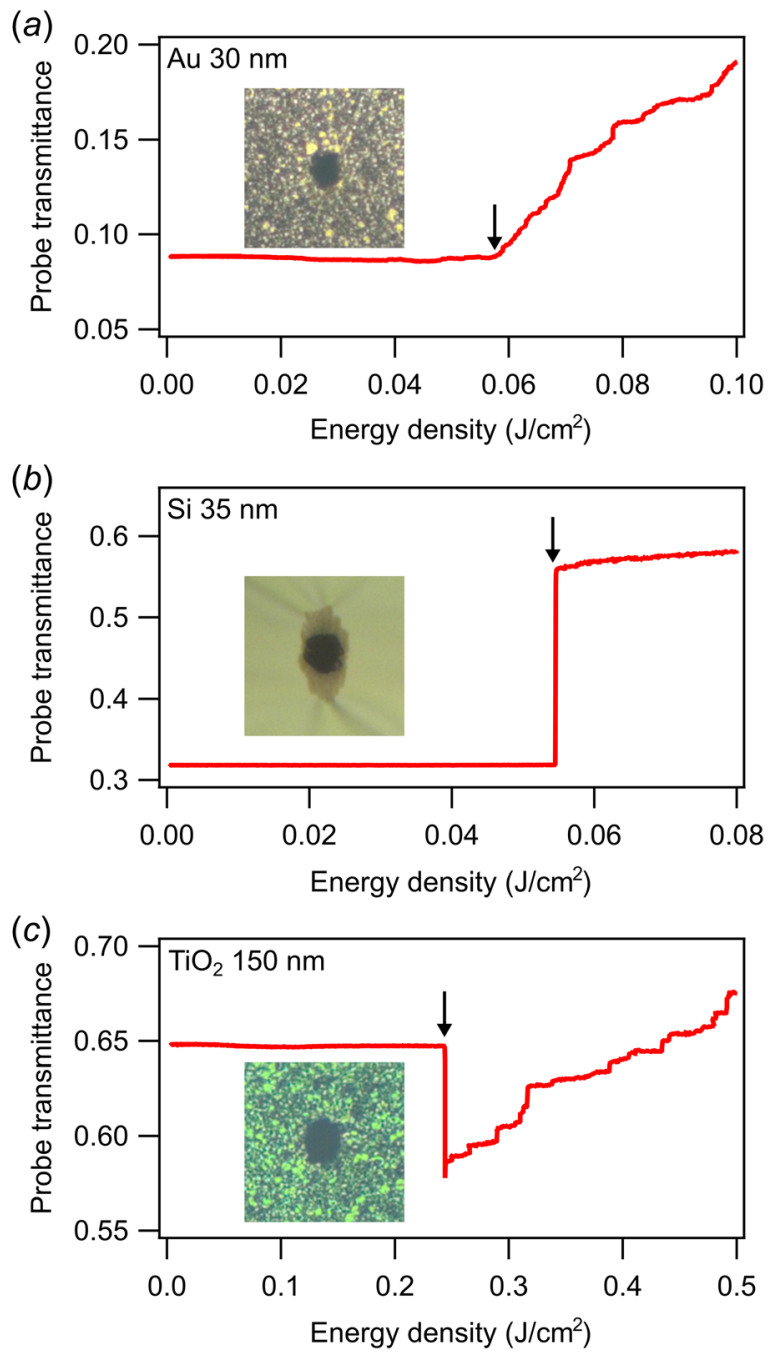

FIG. 2. Laser-damage of free-standing nanometer-thick membranes. (a) Laser-induced damage measurements on 30-nm-thick Au. (b) Laser damage scan of 35-nm-thick Si. (c) Laser damage scan of 150-nm-thick $\mathrm{TiO}_{2}$. Arrows in the figures denote the measured thresholds (see text). Insets, optical dark field microscopy images $\left(100 \times 100 \mu \mathrm{m}^{2}\right)$ of the membranes after the damage scans. Dark spots indicate the holes. signal increase seen in $\mathrm{Si}$ indicates that the initial damage in the center of the laser spot rapidly led to macroscopic damage with a size covering the entire probe beam. Such a sharp increase was also seen in the result of the AlN membrane. On the other hand, the transmittance of the $\mathrm{TiO}_{2}$ membrane showed a drop in probe transmittance. This decrease indicates a change in morphology or chemical composition, for example, partial melting, vaporization, sublimation or crack formation, or alternatively/simultaneously a change in the refractive index or absorption due to a phase transition, oxidation, or other chemical process of the material with air. A similar decrease was also seen for $\mathrm{Al}_{2} \mathrm{O}_{3}$. At powers exceeding the drop threshold, the transmittance started to increase again, probably due to the subsequent formation and further expansion of a macroscopic hole. In view of these observations, a proper definition of damage threshold in the context of this work is the laser fluence (or energy density), at which the probe-beam transmittance starts substantially increasing or decreasing for the first time. The measured laser damage thresholds $\left(F_{50 \mathrm{KHz}}\right)$ are summarized in Table I. These values are the average of three results, each measured with fresh samples. In the analysis of the laser damage threshold of the dielectric or semiconductor membranes, we took into account the Fresnel reflections on the membrane surfaces and the interference of the laser electric fields inside the membranes. $^{31}$ The reported damage values are therefore maximum peak fields/fluences inside the material.

Based on a 1-dimensional thermal diffusion model, ${ }^{27}$ the thermal relaxation time in our foils is microseconds or longer. Repetitive heat deposition can therefore become significant at laser repetition rates in the $\mathrm{kHz}$ regime. In order to investigate the effect of the average power or the number of laser shots on the laser damage, we repeated the measurements by using a fast optical chopper which selectively transmitted only 4 or 5 successive laser pulses at about one tenth $(550 \mathrm{~Hz})$ of the basic laser repetition rate; see Fig. 1(c). The number of laser shots and the average power on the sample were therefore only $5 \%$ of the above measurements, but the pulse energy and the electric peak field strength remained the same. The results $\left(F_{\text {burst }}\right)$ are shown in Table I. These values are similar to those measured without the chopper $\left(F_{50 \mathrm{KHz}}\right)$, suggesting that laser damage with the applied 1-ps pulses is rather exclusively determined by the pulse energy or peak field, and not by the average power or heating effects. It can be concluded that all investigated nano-membranes do not substantially suffer from accumulated heating due to their nanometer thickness. Indeed, damage at any of the investigated repetition rates (up to $50 \mathrm{kHz}$ ) seems dominated by a mechanism that primarily depends on the single-pulse fluence or single-pulse peak field strength.

Interestingly, there are some residual deviations from this overall picture. The ratio $F_{\text {burst }} / F_{50 \mathrm{kHz}}$ is a little larger than one for most of the membranes. It has indeed been seen before that multi-shot illumination can reduce the laser damage threshold by incubation effects, ${ }^{32}$ for example, by the formation of laser-induced defects and nano-crackings ${ }^{33}$ or by thermal accumulation. ${ }^{34}$ The incubation effect usually saturates at a large number of laser shots, typically $10^{4}, 33$ which is more than two orders-of-magnitude lower than in 
our experiment. It is therefore likely that the slightly lower damage threshold at $50 \mathrm{kHz}$ is related to a slightly elevated temperature in the laser spot via the associated mechanical stress, although the main mechanism remains to be a singlepulse effect or high-field damage by the laser's electric field.

We next compared the damage thresholds of freestanding nanometer membranes with those of bulk materials. For this purpose, we measured some damage thresholds of thick transparent materials, namely for single-crystalline $\mathrm{Al}_{2} \mathrm{O}_{3}$ with $200 \mu$ m thickness and (0001) orientation, for $\mathrm{SiO}_{2}$ in $(11 \overline{2} 0)$ orientation with $200 \mu \mathrm{m}$ thickness and for $\mathrm{TiO}_{2}$ in (100) orientation with $500 \mu \mathrm{m}$ thickness (all MTI, Inc.). The laser spot sizes were $27 \times 28 \mu \mathrm{m}^{2}$ for $\mathrm{TiO}_{2}$ and $18 \times 50 \mu \mathrm{m}^{2}$ for $\mathrm{Al}_{2} \mathrm{O}_{3}$ and $\mathrm{SiO}_{2}$, while all other laser parameters were nearly identical to those employed in the membrane experiments. In the analysis of damage thresholds of bulk materials, we considered the electric field amplitudes on the rear surface, where there is constructive optical interference. The measured bulk damage thresholds were $1.2 \pm 0.3,1.8 \pm 0.4$, and $0.20 \pm 0.04 \mathrm{~J} / \mathrm{cm}^{2}$ for $\mathrm{Al}_{2} \mathrm{O}_{3}, \mathrm{SiO}_{2}$, and $\mathrm{TiO}_{2}$, respectively. These damage thresholds are different from those in the nanometer membranes by less than a factor of two, although the thicknesses were up to 5000 times larger.

\section{DISCUSSION}

Table I reveals an increase of damage thresholds with materials' band gap. This band gap dependence can be qualitatively explained by differences in laser photo-absorption mechanisms: ${ }^{31}$ at excitation pulse durations of around 1-ps, which are short enough to decouple the optical absorption and from slower thermal relaxation, ${ }^{22}$ the ultrafast physics of photo-absorption becomes dominant over mechanical or temperature effects. Therefore, the absorbed energy dictates whether a foil is damaged. ${ }^{35}$

Metals ( $\mathrm{Au}$ and $\mathrm{Cu}$ in this work) directly absorb the laser excitation, and one-photon absorption is also the predominant mechanism in graphene at intensities applied in this work. ${ }^{36,37}$ Dielectrics/semiconductors are, in principle, nonabsorbing, but multi-photon effects, defects, tunneling processes, and avalanche ionization create considerable free carriers in the case of strong-field/short-pulse excitation. ${ }^{38}$ When a carrier density reaches a critical value of typically $10^{21} \mathrm{~cm}^{-3}$, where the plasma wave is resonant to the laser frequency, dielectrics/semiconductors can become strongly absorptive, leading to damage. ${ }^{31,39}$ For narrow band-gap materials (Ge and $\mathrm{Si}$ ), predominantly, one-photon or twophoton absorption leads to the critical density. ${ }^{35}$ For wideband-gap materials ( $\mathrm{AlN}, \mathrm{Al}_{2} \mathrm{O}_{3}$, formvar, $\mathrm{Si}_{3} \mathrm{~N}_{4}, \mathrm{SiO}_{2}$, and $\mathrm{TiO}_{2}$ ), some free-electrons are first generated through multiphoton/tunneling ionization or from defects and subsequently multiplied rapidly through avalanche processes, in which free carriers absorb photons and impact-ionize valence electrons. Avalanche ionization is expected to be rather efficient at 1-ps pulse duration. ${ }^{22,38}$ Keldysh theory $^{25,40}$ predicts that the tunneling and multiphoton ionization rates decay almost exponentially with the band gap, suggesting that wider-band-gap materials have higher damage thresholds, as observed.
A highly important excitation parameter for most solidstate attosecond experiments, ${ }^{2-8}$ or when one aims at seeing atomic-scale carrier motions by electron diffraction, ${ }^{21,41,42}$ is the maximum applicable electric peak field, because interesting strong-field effects can occur if the excitation field approaches the threshold where the inter-band tunneling becomes significant. Figure 3 (open circles) shows for all the investigated membranes the peak field-strength at the damage threshold as a function of a material's band gap. ${ }^{43-48}$ We plot the fields inside the material here, not the incoming field strengths. As is already evident from Table I, there is a clear increase of the applicable peak field strength with increasing band gap. This trend qualitatively agrees with the results of bulk materials ${ }^{49,50}$ and optical coatings on substrates. ${ }^{31,35,51,52}$ Only the damage threshold of the AlN membrane is somewhat lower than expected, even though it has the highest damage threshold among the semiconducting materials used in this study. It is probably because the membrane size of $40 \mu \mathrm{m}$ was not substantially larger than the excitation spot, and the outer part of the laser could have ablated the silicon substrate which has a lower threshold. The ablation of the silicon substrate is indeed seen in optical microscopy images after the scans. The fact that our nanometer-thick membranes with their unavoidable impurities and defects are revealing this band gap correlation effect is a clear sign that the underlying physics of laser damage is approximately independent of the sample thickness. Therefore, high-field studies by attosecond spectroscopy or ultrafast electron diffraction can rely on nanometer-thick membranes without substantial danger of creating distortions via size effects.

For several high-band-gap materials, the available field strength with our 1 -ps pulses exceeded $1 \mathrm{GV} / \mathrm{m}$, which is high enough to investigate light-field-driven electronic processes by attosecond spectroscopy ${ }^{2-4,53}$ or space-time ultrafast electron diffraction. ${ }^{21}$ If field strengths approaching the inter-atomic dimensions of $\sim 1 \mathrm{~V} / \AA$ are required, shorter pulses should be applied. ${ }^{2,3,5,36}$ By invoking the empirical scaling law $F \propto \tau^{0.3},{ }^{31}$ where $F$ is the damage threshold fluence and $\tau$ is the pulse duration, the applicable peak field strength scales with $\tau^{-0.35}$, i.e., shorter pulses provide higher

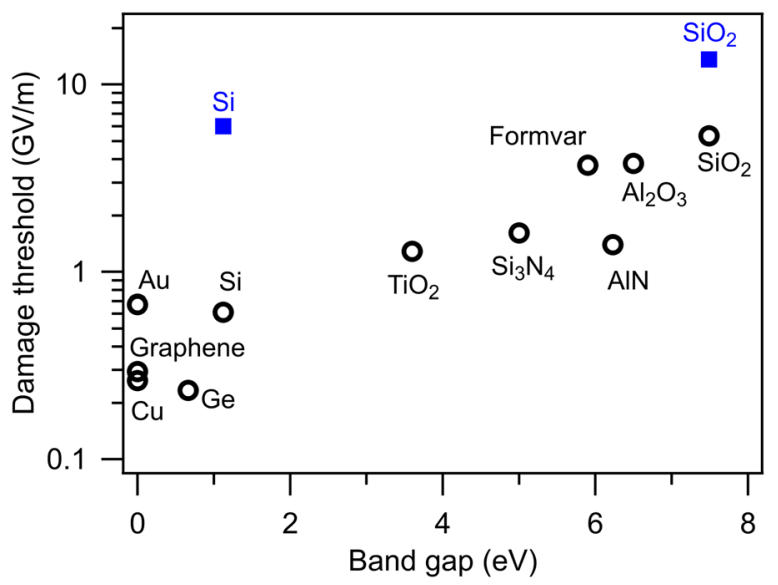

FIG. 3. Applicable peak electric field in free-standing nanometer membranes as a function of band gap. Open circles, experimental results of this work obtained with 1-ps pulses. Squares, damage thresholds measured with few-cycle pulses at $800 \mathrm{~nm}$. 
fields. This estimation suggests that the maximum field strength that is applicable to our nanometer-thick membranes can reach the level of $1 \mathrm{~V} / \AA$, when single-cycle or few-cycle optical pulses are applied. The squares in Fig. 3 show two literature values for no damage with few-cycle excitation., ${ }^{3,5}$ Indeed, damage occurs at a substantially higher field strength, as expected.

\section{CONCLUSION}

In summary, our investigation of laser damage of freestanding nanometer-thin membranes made of 11 different materials reveals the thickness-independence of damage and its increase with band gap. Although the membranes are ultrathin, laser damage does not significantly depend on the repetition rate in the $\mathrm{kHz}$ regime, and the damage mechanism is predominantly due to peak field strength and fluence. A peak field greater than $1 \mathrm{GV} / \mathrm{m}$ can be applied to many materials even with rather long 1-ps pulses; shorter excitation pulses can produce inter-atomic field strengths (approaching $1 \mathrm{~V} / \AA$ ). Therefore, nanometer-thick membranes are well suited for high-field/attosecond investigations of band structure dynamics or for atomic-resolution space-time imaging by electron diffraction/microscopy in transmission geometry.

\section{ACKNOWLEDGMENTS}

This work was supported by the European Research Council and the Munich-Centre for Advanced Photonics. Y.M. acknowledges support from a JSPS Postdoctoral Fellowship for Research Abroad. We thank Ferenc Krausz for support, Simon Stork for preparing $\mathrm{Al}_{2} \mathrm{O}_{3}, \mathrm{Au}, \mathrm{Cu}, \mathrm{Ge}$, and $\mathrm{TiO}_{2}$ membranes, and Artur Engelmann and Tobias Boolakee for assistance in preliminary experiments.

${ }^{1}$ Y. Morimoto and P. Baum, "Diffraction and microscopy with attosecond electron pulse trains," Nat. Phys. (published online).

${ }^{2}$ M. Schultze, E. M. Bothschafter, A. Sommer, S. Holzner, W. Schweinberger, M. Fiess, M. Hofstetter, R. Kienberger, V. Apalkov, V. S. Yakovlev, M. I. Stockman, and F. Krausz, "Controlling dielectrics with the electric field of light," Nature 493(7430), 75-78 (2013).

${ }^{3}$ M. Schultze, K. Ramasesha, C. D. Pemmaraju, S. A. Sato, D. Whitmore, A. Gandman, J. S. Prell, L. J. Borja, D. Prendergast, K. Yabana, D. M. Neumark, and S. R. Leone, "Attosecond band-gap dynamics in silicon," Science 346(6215), 1348-1352 (2014).

${ }^{4}$ M. Lucchini, S. A. Sato, A. Ludwig, J. Herrmann, M. Volkov, L. Kasmi, Y. Shinohara, K. Yabana, L. Gallmann, and U. Keller, "Attosecond dynamical Franz-Keldysh effect in polycrystalline diamond," Science 353(6302), 916-919 (2016).

${ }^{5}$ T. T. Luu, M. Garg, S. Y. Kruchinin, A. Moulet, M. T. Hassan, and E. Goulielmakis, "Extreme ultraviolet high-harmonic spectroscopy of solids," Nature 521(7553), 498-502 (2015).

${ }^{6}$ G. Vampa, T. J. Hammond, N. Thiré, B. E. Schmidt, F. Légaré, C. R. McDonald, T. Brabec, and P. B. Corkum, "Linking high harmonics from gases and solids," Nature 522(7557), 462-464 (2015).

${ }^{7}$ H. Liu, Y. Li, Y. S. You, S. Ghimire, T. F. Heinz, and D. A. Reis, "Highharmonic generation from an atomically thin semiconductor," Nat. Phys. 13, 262-265 (2017).

${ }^{8}$ G. Ndabashimiye, S. Ghimire, M. X. Wu, D. A. Browne, K. J. Schafer, M. B. Gaarde, and D. A. Reis, "Solid-state harmonics beyond the atomic limit," Nature 534(7608), 520-523 (2016).

${ }^{9}$ K. E. Priebe, C. Rathje, S. V. Yalunin, T. Hohage, A. Feist, S. Schäfer, and C. Ropers, "Attosecond electron pulse trains and quantum state reconstruction in ultrafast transmission electron microscopy," arXiv:1706.03680.
${ }^{10}$ A. H. Zewail, "Four-dimensional electron microscopy," Science 328(5975), 187-193 (2010).

${ }^{11}$ G. Sciaini and R. J. D. Miller, "Femtosecond electron diffraction: Heralding the era of atomically resolved dynamics," Rep. Prog. Phys. 74(9), 096101 (2011).

${ }^{12}$ A. Feist, N. Bach, N. Rubiano da Silva, T. Danz, M. Möller, K. E. Priebe, T. Domröse, J. G. Gatzmann, S. Rost, J. Schauss, S. Strauch, R. Bormann, M. Sivis, S. Schäfer, and C. Ropers, "Ultrafast transmission electron microscopy using a laser-driven field emitter: Femtosecond resolution with a high coherence electron beam," Ultramicroscopy 176, 63-73 (2017).

${ }^{13}$ B. J. Siwick, J. R. Dwyer, R. E. Jordan, and R. J. D. Miller, "An atomiclevel view of melting using femtosecond electron diffraction," Science 302(5649), 1382-1385 (2003).

${ }^{14}$ P. Baum, D. S. Yang, and A. H. Zewail, "4D visualization of transitional structures in phase transformations by electron diffraction," Science 318(5851), 788-792 (2007).

${ }^{15}$ A. Yurtsever, R. M. van der Veen, and A. H. Zewail, "Subparticle ultrafast spectrum imaging in 4D electron microscopy," Science 335(6064), 59-64 (2012).

${ }^{16}$ S. Lahme, C. Kealhofer, F. Krausz, and P. Baum, "Femtosecond singleelectron diffraction," Struct. Dyn. 1(3), 034303 (2014).

${ }^{17}$ T. Ishikawa, S. A. Hayes, S. Keskin, G. Corthey, M. Hada, K. Pichugin, A. Marx, J. Hirscht, K. Shionuma, K. Onda, Y. Okimoto, S. Koshihara, T. Yamamoto, H. B. Cui, M. Nomura, Y. Oshima, M. Abdel-Jawad, R. Kato, and R. J. D. Miller, "Direct observation of collective modes coupled to molecular orbital-driven charge transfer," Science 350(6267), 1501-1505 (2015).

${ }^{18}$ A. Ryabov and P. Baum, "Electron microscopy of electromagnetic waveforms," Science 353(6297), 374-377 (2016).

${ }^{19}$ T. Frigge, B. Hafke, T. Witte, B. Krenzer, C. Streubuhr, A. S. Syed, V. M. Trontl, I. Avigo, P. Zhou, M. Ligges, D. von der Linde, U. Bovensiepen, M. Horn-von Hoegen, S. Wippermann, A. Lücke, U. Gerstmann, and W. G. Schmidt, "Optically excited structural transition in atomic wires on surfaces at the quantum limit," Nature 544(7649), 207-211 (2017).

${ }^{20}$ C. Kealhofer, W. Schneider, D. Ehberger, A. Ryabov, F. Krausz, and P. Baum, "All-optical control and metrology of electron pulses," Science 352(6284), 429-433 (2016).

${ }^{21}$ V. S. Yakovlev, M. I. Stockman, F. Krausz, and P. Baum, "Atomic-scale diffractive imaging of sub-cycle electron dynamics in condensed matter," Sci. Rep. 5, 14581 (2015).

${ }^{22}$ C. B. Schaffer, A. Brodeur, and E. Mazur, "Laser-induced breakdown and damage in bulk transparent materials induced by tightly focused femtosecond laser pulses," Meas. Sci. Technol. 12(11), 1784-1794 (2001).

${ }^{23}$ S. S. Mao, F. Quéré, S. Guizard, X. Mao, R. E. Russo, G. Petite, and P. Martin, "Dynamics of femtosecond laser interactions with dielectrics," Appl. Phys. A 79(7), 1695-1709 (2004).

${ }^{24}$ R. M. Wood, Laser-Induced Damage of Optical Materials, Series in Optics and Optoelectronics (CRC Press, 2003).

${ }^{25} \mathrm{P}$. Balling and J. Schou, "Femtosecond-laser ablation dynamics of dielectrics: Basics and applications for thin films," Rep. Prog. Phys. 76(3), 036502 (2013).

${ }^{26}$ A. A. Manenkov, "Fundamental mechanisms of laser-induced damage in optical materials: Today's state of understanding and problems," Opt. Eng. 53(1), 010901 (2014).

${ }^{27} \mathrm{X}$. Zhang and C. P. Grigoropoulos, "Thermal conductivity and diffusivity of free-standing silicon nitride thin films," Rev. Sci. Instrum. 66(2), 1115-1120 (1995).

${ }^{28}$ W. Schneider, A. Ryabov, C. Lombosi, T. Metzger, Z. Major, J. A. Fülöp, and P. Baum, " $800-\mathrm{fs}, 330-\mu \mathrm{J}$ pulses from a 100-W regenerative Yb:YAG thin-disk amplifier at $300 \mathrm{kHz}$ and $\mathrm{THz}$ generation in $\mathrm{LiNbO}_{3}$," Opt. Lett. 39(23), 6604-6607 (2014).

${ }^{29}$ D. Néel, S. Sergent, M. Mexis, D. Sam-Giao, T. Guillet, C. Brimont, T. Bretagnon, F. Semond, B. Gayral, S. David, X. Checoury, and P. Boucaud, "AlN photonic crystal nanocavities realized by epitaxial conformal growth on nanopatterned silicon substrate," Appl. Phys. Lett. 98(26), 261106 (2011).

${ }^{30}$ F. O. Kirchner, S. Lahme, F. Krausz, and P. Baum, "Coherence of femtosecond single electrons exceeds biomolecular dimensions," New J. Phys. 15(6), 063021 (2013).

${ }^{31}$ M. Mero, J. Liu, W. Rudolph, D. Ristau, and K. Starke, "Scaling laws of femtosecond laser pulse induced breakdown in oxide films," Phys. Rev. B 71(11), 115109 (2005). 
${ }^{32}$ M. Lenzner, J. Krüger, W. Kautek, and F. Krausz, "Incubation of laser ablation in fused silica with 5-fs pulses," Appl. Phys. A: Mater. Sci. Process. 69(4), 465-466 (1999).

${ }^{33}$ Z. Sun, M. Lenzner, and W. Rudolph, "Generic incubation law for laser damage and ablation thresholds," J. Appl. Phys. 117(7), 073102 (2015).

${ }^{34}$ B. J. Nagy, L. Gallais, L. Vámos, D. Oszetzky, P. Rácz, and P. Dombi, "Direct comparison of kilohertz- and megahertz-repetition-rate femtosecond damage threshold," Opt. Lett. 40(11), 2525-2528 (2015).

${ }^{35}$ L. Gallais, D.-B. Douti, M. Commandré, G. Bataviciute, E. Pupka, M. Šciuka, L. Smalakys, V. Sirutkaitis, and A. Melninkaitis, "Wavelength dependence of femtosecond laser-induced damage threshold of optical materials," J. Appl. Phys. 117(22), 223103 (2015).

${ }^{36}$ M. Currie, J. D. Caldwell, F. J. Bezares, J. Robinson, T. Anderson, H. Chun, and M. Tadjer, "Quantifying pulsed laser induced damage to graphene," Appl. Phys. Lett. 99(21), 211909 (2011).

${ }^{37}$ G. K. Lim, Z. L. Chen, J. Clark, R. G. S. Goh, W. H. Ng, H. W. Tan, R. H. Friend, P. K. H. Ho, and L. L. Chua, "Giant broadband nonlinear optical absorption response in dispersed graphene single sheets," Nat. Photonics 5(9), 554-560 (2011)

${ }^{38}$ B. C. Stuart, M. D. Feit, A. M. Rubenchik, B. W. Shore, and M. D. Perry, "Laser-induced damage in dielectrics with nanosecond to subpicosecond pulses," Phys. Rev. Lett. 74(12), 2248-2251 (1995).

${ }^{39}$ B. C. Stuart, M. D. Feit, S. Herman, A. M. Rubenchik, B. W. Shore, and M. D. Perry, "Nanosecond-to-femtosecond laser-induced breakdown in dielectrics," Phys. Rev. B 53(4), 1749-1761 (1996).

${ }^{40} \mathrm{~L}$. V. Keldysh, "Ionization in the field of a strong electromagnetic wave," Sov. Phys. JETP 20(5), 1307 (1965).

${ }^{41} \mathrm{P}$. Baum, "Towards ultimate temporal and spatial resolutions with ultrafast single-electron diffraction," J. Phys. B: At. Mol. Opt. Phys. 47(12), 124005 (2014).

${ }^{42}$ H. C. Shao and A. F. Starace, "Detecting electron motion in atoms and molecules," Phys. Rev. Lett. 105(26), 263201 (2010).

${ }^{43}$ T. M. Donovan, W. E. Spicer, J. M. Bennett, and E. J. Ashley, "Optical properties of amorphous germanium films,” Phys. Rev. B 2(2), 397-413 (1970).
${ }^{44}$ A. R. Forman, W. R. Thurber, and D. E. Aspnes, "Second indirect band gap in silicon," Solid State Commun. 14(10), 1007-1010 (1974).

${ }^{45} \mathrm{~L}$. Gallais and M. Commandré, "Laser-induced damage thresholds of bulk and coating optical materials at $1030 \mathrm{~nm}, 500 \mathrm{fs}$," Appl. Opt. 53(4), A186-A196 (2014).

${ }^{46}$ A. R. Forouhi and I. Bloomer, "Optical dispersion relations for amorphous semiconductors and amorphous dielectrics," Phys. Rev. B 34(10), 7018-7026 (1986)

${ }^{47}$ W. M. Yim, E. J. Stofko, P. J. Zanzucchi, J. I. Pankove, M. Ettenberg, and S. L. Gilbert, "Epitaxially grown AlN and its optical band gap," J. Appl. Phys. 44(1), 292-296 (1973).

${ }^{48}$ O. G. Abdullah, S. B. Aziz, K. M. Omer, and Y. M. Salih, "Reducing the optical band gap of polyvinyl alcohol (PVA) based nanocomposite," J. Mater. Sci.: Mater. Electron. 26(7), 5303-5309 (2015).

${ }^{49}$ D. M. Simanovskii, H. A. Schwettman, H. Lee, and A. J. Welch, "Midinfrared optical breakdown in transparent dielectrics," Phys. Rev. Lett. 91(10), 107601 (2003).

${ }^{50}$ D. Grojo, S. Leyder, P. Delaporte, W. Marine, M. Sentis, and O. Utéza, "Long-wavelength multiphoton ionization inside band-gap solids," Phys. Rev. B 88(19), 195135 (2013).

${ }^{51}$ B. Mangote, L. Gallais, M. Commandré, M. Mende, L. Jensen, H. Ehlers, M. Jupé, D. Ristau, A. Melninkaitis, J. Mirauskas, V. Sirutkaitis, S. Kicas, T. Tolenis, and R. Drazdys, "Femtosecond laser damage resistance of oxide and mixture oxide optical coatings," Opt. Lett. 37(9), $1478-1480$ (2012)

${ }^{52}$ I. B. Angelov, M. Pechmann, M. K. Trubetskov, F. Krausz, and V. Pervak, "Optical breakdown of multilayer thin-films induced by ultrashort pulses at MHz repetition rates," Opt. Express 21(25), 31453-31461 (2013).

${ }^{53}$ A. Sommer, E. M. Bothschafter, S. A. Sato, C. Jakubeit, T. Latka, O. Razskazovskaya, H. Fattahi, M. Jobst, W. Schweinberger, V. Shirvanyan, V. S. Yakovlev, R. Kienberger, K. Yabana, N. Karpowicz, M. Schultze, and F. Krausz, "Attosecond nonlinear polarization and lightmatter energy transfer in solids," Nature 534(7605), 86-90 (2016). 